\title{
Índice de Massa Corpórea e circunferência abdominal como preditores de risco cardiovascular em pacientes diabéticos
}

\author{
Body Mass Index and waist circumference as predictors of cardiovascular risk in diabetic patients \\ Índice de Masa Corporal y circunferencia abdominal como predictores de riesgo cardiovascular en \\ pacientes diabéticos
}

Recebido: 16/10/2021 | Revisado: 27/10/2021 | Aceito: 30/10/2021 | Publicado: 01/11/2021

\author{
Nathalie Macêdo Cruz de Oliveira \\ ORCID: https://orcid.org/0000-0003-2321-8486 \\ Universidade Federal de Alagoas, Brasil \\ E-mail: nathaliemacedo2@gmail.com \\ José Ayrton Macedo Guimarães de Oliveira \\ ORCID: https://orcid.org/0000-0003-3180-6309 \\ Universidade Estadual de Ciências da Saúde de Alagoas, Brasil \\ E-mail: ayrtonmacedo5@gmail.com \\ André Falcão Pedrosa Costa \\ ORCID: https://orcid.org/0000-0002-2286-7946 \\ Universidade Estadual de Ciências da Saúde de Alagoas, Brasil \\ E-mail: falcaopedrosa@uol.com.br
}

\begin{abstract}
Resumo
Uma condição altamente prevalente em diabéticos é obesidade. O objetivo foi observar dos métodos de avaliação de obesidade: IMC e circunferência abdominal (CA) e verificar sua associação com fatores de risco cardiovascular (FRCV) em diabéticos. Através de estudo prospectivo e transversal foram avaliados diabéticos atendidos em uma Atenção Primaria. Foram medidos pesos e estatura para calcular IMC; CA e de quadril para relação cintura/quadril. Bioquímica, bem como dados demográficos foram coletados de prontuários para análise clínica. Pressão arterial medida na avaliação. Utilizou Bioestat 5.0 para estadística. As prevalências de sobrepeso e obesidade foram elevadas bem como a de FRCV, incluindo CA. Prevalência de síndrome metabólica (SM) foi de 67,1\%. Não houve associação entre FRCV e antropometria. CA foi capaz de predizer SM. Se recomenda utilizar CA em prática clínica, mas não como parâmetro clínico único, visto a possibilidade de diagnóstico incompleto e possível atraso em medidas terapêuticas.
\end{abstract}

Palavras-chave: Diabetes mellitus; Síndrome metabólica; Obesidade; Índice de massa corporal; Circunferência abdominal.

\begin{abstract}
Obesity is a highly prevalent condition in diabetics. The aim was to observe two methods of obesity evaluation: BMI and abdominal circumference (AC) and its association with cardiovascular risk factors in diabetics. Through a prospective, cross-sectional study, diabetics seen in primary care were assessed. Body weight and height were measured to calculate BMI; AC and hip circumference for waist / hip ratio. Biochemistry, well as demographic data were collected from records for clinical analysis. Blood pressure measured at the evaluation. Using BioStat 5.0 for statistical. Prevalence of overweight and obesity were high as the prevalence of cardiovascular risk factors, included AC. Prevalence of Metabolic Syndrome (MS) was 67.1\%. There was no association between the risk factors and anthropometry. AC was able to predict SM. It is recommended to use the AC in clinical practice, but not as unique clinical parameter, since the possibility of misdiagnosis and treatment delay.
\end{abstract}

Keywords: Diabetes mellitus; Metabolic syndrome; Obesity; Body mass index; Abdominal circumference.

\section{Resumen}

Una condición altamente prevalente en diabéticos es obesidad. El objetivo fue observar dos métodos de avaluación de obesidad: IMC y circunferencia abdominal (CA) y verificar su asociación con factores de riesgo cardiovascular (FRCV) en diabéticos. A través de estudio prospectivo y transversal fueron avaluados diabéticos atendidos en Atención Primaria. Fueron medidos peso y estatura para calcular IMC; CA y de cadera para relación cintura/cadera. Bioquímica, bien como datos demográficos fueron coleccionados de registros para análisis clínica. Presión arterial medida en la avaluación. Utilizó Bioestat 5.0 para estadística. Las prevalencias de sobrepeso y obesidad fueron elevadas bien como la de FRCV, incluyendo CA. Prevalencia de Síndrome Metabólico (SM) fue de 67,1\%. No hubo asociación entre FRCV y antropometría. CA fue capaz de predecir SM. Se recomienda utilizar CA en práctica clínica, 
pero no como parámetro clínico único, visto la posibilidad de diagnóstico incompleto y posible atraso en medidas terapéuticas.

Palabras clave: Diabetes mellitus; Síndrome metabólico; Obesidad; Índice de masa corporal; Circunferencia abdominal.

\section{Introdução}

A diabetes mellitus (DM) é a desordem metabólica mais comum no mundo, estimada em mais de 171 milhões de pessoas (Castro et al., 2006; Ginsberg et al., 2009). Os pacientes com DM apresentam frequentemente hiperglicemia, pressão arterial elevada, hipertrigliceridemia, níveis baixos de lipoproteína de alta densidade (HDL-c) e obesidade central, que são fatores de risco para enfermidade cardiovascular e principal causa de morte global (Stolar \& Chilton, 2003).

De acordo com o National Cholesterol Education Program - Adult Treatment Panel III (NCEP - ATP III), o grupo onde esses fatores coexistem é definido como síndrome metabólica (SM), uma condição com maior risco de complicações na DM (Castro et al., 2006; Gierach et al., 2014).

A adiposidade corporal provou ser um importante fator de risco para a enfermidade cardiovascular e é fortemente associada a fatores de risco para enfermidade cardiovascular (Van Dijk et al., 2012). Medidas antropométricas são rotineiramente empregadas para sua avaliação. A circunferência abdominal (CA) e a relação cintura-quadril (RCQ) são utilizadas como medidas regionais de obesidade e o índice de massa corporal (IMC) como medida geral de obesidade (Rocha et al., 2010). Porém, a maioria dos estudos diverge sobre o melhor preditor antropométrico de risco cardiovascular e SM.

As consequências que a modificação da composição corporal pode causar na situação nutricional na saúde estão já estabelecidas. No entanto, a adoção de um modelo único de classificação nutricional pode ocasionar avaliações imprecisas e um diagnóstico incorreto (Glaner, 2005; Martins et al., 2011). O objetivo do estudo foi observar o método antropométrico ou a relação mais eficaz na avaliação dos pacientes diabéticos, sua associação com fatores de risco para enfermidade cardiovascular e a presença de SM em uma população de diabéticos atendidos na atenção primaria onde os estudos são escassos em países latino-americanos.

\section{Metodologia}

A pesquisa foi realizada em uma Unidade de Saúde da Família no período de agosto de 2013 até julho de 2014. Se trata de um estudo de caráter clínico prospectivo, transversal, sem técnicas de mascaramento, no qual foram incluídos indivíduos diabéticos tipo 2, que depois de informados sobre a pesquisa, concordaram em participar e assinaram um termo de consentimento livre e esclarecido. Setenta e seis pessoas foram incluídas na pesquisa.

Todos os pacientes foram submetidos a una análise nutricional, onde foram coletadas medidas de peso, altura, CA e circunferência do quadril (CQ). Os dados antropométricos como peso (em $\mathrm{Kg}$ ) e tamanho (em M), serviram para o cálculo do IMC e foram obtidos pelo mesmo examinador usando uma mesma balança previamente calibrada. A CA em centímetros, definida como a menor curvatura entre medida de uma circunferência no nível da cicatriz do umbigo, no final do movimento expiratório e a CQ em centímetros, definida como a maior medida de uma circunferência no nível do quadril e nádegas, foram obtidas através de fita métrica flexível para o cálculo da RCQ obtido pela razão entre CA e CQ e serviram para predizer a gordura visceral e parâmetro para SM.

Em seguida, para a análise clínica foram coletados pelos registros médicos dos pacientes, dados disponíveis como exames bioquímicos: glicemia de jejum, hemoglobina glicada, colesterol total e frações e triglicerídeos. Além do mais, os dados demográficos como sexo (categorizado em masculino e feminino) e idade (em anos).

Posteriormente, os indivíduos foram submetidos a medição da pressão arterial segundo modelo auscultatório de Korotkoff. Foram definidos como fatores de risco para enfermidade cardiovascular: CA $\geq 80 \mathrm{~cm}$ no sexo feminino e $\geq 94 \mathrm{~cm}$ 
no sexo masculino, valores de triglicerídeos $\geq 150 \mathrm{mg} / \mathrm{dl}$, valores de glicemia $\geq 100 \mathrm{mg} / \mathrm{dl}$, níveis de colesterol total $\geq 200$ $\mathrm{mg} / \mathrm{dl}$, níveis de lipoproteína de baixa densidade (LDL-c) $\geq 130 \mathrm{mg} / \mathrm{dl}$ e níveis de $\mathrm{HDL}<40 \mathrm{mg} / \mathrm{dl}$ para homens e $<50 \mathrm{mg} / \mathrm{dl}$ para mulheres, valores de pressão arterial sistólica (PAS) $\geq 130 \mathrm{mmHg}$ e de pressão arterial diastólica (PAD) $\geq 85 \mathrm{mmHg}$.

E a presença de SM foi definida de acordo com os critérios da American Heart Association (AHA), em qual requer a presença de três ou mais dos cinco fatores que se seguem: a) obesidade abdominal: quando a CA seja maior que $88 \mathrm{~cm} \mathrm{em}$ mulheres e maior que $102 \mathrm{~cm}$ em homens; b) concentração plasmática de triglicerídeos $\geq 150 \mathrm{mg} / \mathrm{dl}$; c) concentração plasmática de HDL-c menor que $40 \mathrm{mg} / \mathrm{dl}$ para homens e $50 \mathrm{mg} / \mathrm{dl}$ para mulheres; d) PAS $\geq 130 \mathrm{mmHg}$ o PAD $\geq 85 \mathrm{mmHg}$; e) glicemia de jejum $\geq 100 \mathrm{mg} / \mathrm{dl}$ (Ginsberg et al., 2009).

A amostra foi aleatória porque se propôs a incluir todos os pacientes, de uma unidade básica de saúde, portadores de DM tipo 2. Todos os dados foram avaliados em forma de média e desvio-padrão quando as variáveis apresentavam distribuição normal ou mediana quando a distribuição não era gaussiana. Todos os cálculos estatísticos foram realizados pelo programa BioEstat 5.0. Para a correlação entre o IMC e a CA, foi utilizado correlações entre variáveis contínuas, usando a correlação de Spearman. Para averiguar a predição de SM foi utilizado o teste exato de Fisher. Para estimativa do desempenho dos métodos antropométricos se usou o Screening test (crivo). Em todos os casos, se admitiu um intervalo de confiança de 95\%.

\section{Resultados}

Entre os 76 indivíduos estudados, $50(65,78 \%)$ eram do sexo feminino e $26(34,21 \%)$ do sexo masculino, com predomínio de idade na faixa de 50 a 59 anos. Avaliando o padrão de IMC, se observou que a prevalência de sobrepeso e obesidade de $34 \%$ e $54 \%$, respectivamente, no sexo feminino, e de $48 \%$ e $36 \%$, respectivamente, no sexo masculino. Com relação ao padrão de circunferência abdominal, $14 \%$ das mulheres apresentaram CA na faixa de 80 a $88 \mathrm{~cm}$ e $80 \%$ das mulheres apresentaram CA acima de $88 \mathrm{~cm}$. Nos homens, $24 \%$ apresentaram CA na faixa de 94 a $102 \mathrm{~cm}$ e $60 \%$ CA acima de $102 \mathrm{~cm}$. Quando comparadas estatisticamente, as duas variáveis apresentaram uma correlação positiva e estatisticamente significativa no sexo feminino (coef. de Spearman $=0,5419$, $\mathrm{p}<0,0001$ vs. $\mathrm{p}=0,9301$ ).

$\mathrm{Na}$ análise clínica, se encontrou uma elevada prevalência dos fatores de risco para enfermidade cardiovascular e critérios da SM, 84,84\% apresentaram glicemia de jejum alterada, 44\% colesterol total elevado, 77\% HDL-c baixo, 46,66\% LDL-c alto, 54,9\% hipertrigliceridemia, 58,90\% níveis de PAS $\geq 130 \mathrm{mmHg}$ e 42,46\% níveis de PAD $\geq 85 \mathrm{mmHg}$. Não houve diferença significativa entre os sexos e a distribuição seguiu conforme a Tabela 1.

Tabela 1. Características antropométrica, bioquímica e clínica de indivíduos diabéticos atendidos na Atenção Primária.

\section{Variáveis}

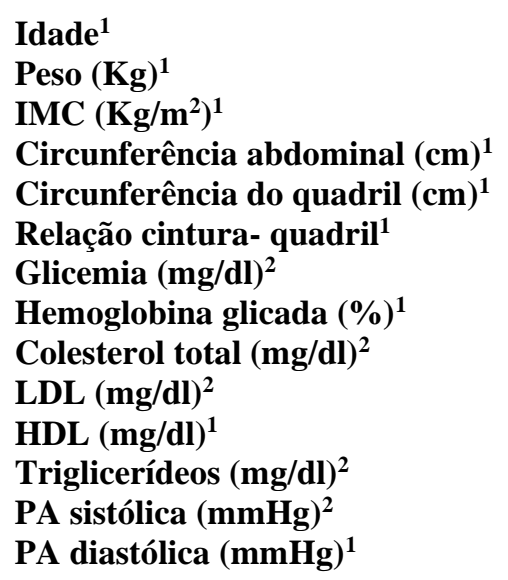

\section{Feminino}

$58,17 \pm 8,58$

$71,24 \pm 14,21$

$30,66 \pm 5,35$

$96,46 \pm 11,27$

$102,5 \pm 11,33$

$0,93 \pm 0,07$

157 (62-394)

$8,38 \pm 1,4$

$202(140-298)$

141 (80-232)

$39,15 \pm 10,51$

200 (99-414)

130 (90-230)

$81,43 \pm 11,35$

\section{Masculino}

$57,96 \pm 9,69$

$78,81 \pm 12,13$

$29,01 \pm 4,06$

$102,48 \pm 9,84$

$105,84 \pm 9,28$

$0,96 \pm 0,07$

168 (76-330)

$9,31 \pm 2,24$

$188(118-293)$

103 (78-177)

$37 \pm 10,53$

$140(35-375)$

139 (110-197)

$83.76 \pm 9,27$

${ }^{1}$ média \pm desvio-padrão; ${ }^{2}$ mediana (mínimo e máximo) Fonte: Autores. 
Comparando as variáveis antropométricas e os fatores de risco para enfermidade cardiovascular se encontrou uma pequena importância estatística e correlação negativa entre as variáveis IMC e PAD como pode ser visto na Tabela 2, a qual não se repetiu quando da análise por quartis.

Tabela 2. Correlação entre variáveis antropométricas e fatores de risco cardiovascular em indivíduos diabéticos de uma unidade de saúde de Alagoas.

\begin{tabular}{llll}
\hline \multicolumn{1}{c}{ Variables } & \multicolumn{1}{c}{ IMC } & \multicolumn{1}{c}{ CA } & RCQ \\
\hline Triglicerídeos & $\mathrm{rs}=-0.1506$ & $\mathrm{rs}=-0.2521$ & $\mathrm{rs}=0.0686$ \\
Glicemia & $\mathrm{P}=0.2915$ & $\mathrm{p}=0.0743$ & $\mathrm{p}=0.6326$ \\
& $\mathrm{rs}=0.0417$ & $\mathrm{rs}=0.0364$ & $\mathrm{rs}=0.0183$ \\
Colesterol Total & $\mathrm{p}=0.7394$ & $\mathrm{p}=0.7714$ & $\mathrm{p}=0.8842$ \\
& $\mathrm{rs}=-0.1905$ & $\mathrm{rs}=-0.2346$ & $\mathrm{rs}=0.1647$ \\
LDL Colesterol & $\mathrm{p}=0.1760$ & $\mathrm{p}=0.0940$ & $\mathrm{p}=0.2433$ \\
& $\mathrm{rs}=-0.0084$ & $\mathrm{rs}=-0.1417$ & $\mathrm{rs}=-0.320$ \\
HDL Colesterol & $\mathrm{p}=0.9656$ & $\mathrm{p}=0.4635$ & $\mathrm{p}=0.8690$ \\
& $\mathrm{rs}=0.2224$ & $\mathrm{rs}=0.0568$ & $\mathrm{rs}=0.1836$ \\
PAS & $\mathrm{p}=0.2648$ & $\mathrm{p}=0.7782$ & $\mathrm{p}=0.3593$ \\
& $\mathrm{rs}=-0.125$ & $\mathrm{rs}=0.1770$ & $\mathrm{rs}=0.0601$ \\
PAD & $\mathrm{P}=0.9163$ & $\mathrm{p}=0.1340$ & $\mathrm{p}=0.6135$ \\
& $\mathrm{rs}=-0.2384$ & $\mathrm{rs}=0.0797$ & $\mathrm{rs}=0.1869$ \\
& $\mathrm{~A} \mathrm{p}=0.0422$ & $\mathrm{p}=0.5025$ & $\mathrm{p}=0.1133$ \\
\hline
\end{tabular}

rs: Correlação de Spearman, ${ }^{A}$ p <0.05; IMC: Índice de Massa Corporal; CA: Circunferência abdominal; RCC: Relação cintura-quadril; PAS: Pressão Arterial Sistólica, PAD: Pressão Arterial Diastólica.

Fonte: Autores.

A prevalência de SM foi de $67,1 \%$. Ao avaliar o IMC e a CA como preditores de SM, a CA apresentou significância estatística ( $<<0,0001$ vs $p=0,4227$ ), sendo sua sensibilidade de $82,35 \%$ e especificidade $100 \%$.

\section{Discussão}

Segundo a Pesquisa de Orçamento Familiar (POF) 2008-2009, o excesso de peso em homens adultos saltou de 18,5\% para 50,1\%, e em mulheres de $28,7 \%$ para $48 \%$. Desses, ao redor de $1 / 3(12,5 \%)$ dos homens e $1 / 3(16,9 \%)$ das mulheres eram obesos (Marinho et al., 2012). Dada sua elevada e crescente prevalência, a obesidade pode ser considerada uma pandemia (Ginsberg et al., 2009). De fato, ao comparar os resultados da pesquisa com um estudo metacêntrico brasileiro em uma população específica semelhante de DM2 do ano de 2006, (Gomes et al., 2006) a prevalência de obesidade também se mostrou superior ao registro de $17,9 \%$ daquele ano na região nordeste.

A obesidade é um fator de risco estabelecido para DM2 e componente central da SM. O armazenamento de depósitos de gordura visceral libera ácidos graxos livres capazes de reduzir a depuração hepática de insulina contribuindo para o desenvolvimento de resistência insulínica e hiperinsulinemia (Kodama et al., 2013). Não surpreende, por tanto, o aumento da prevalência de obesidade ser paralelamente seguido do aumento do número de pessoas com DM2 e SM (Ginsberg et al., 2009). Logo, já era de se esperar uma alta prevalência de SM na amostra, a qual se mostrou até superior as taxas representativas da I Diretriz Brasileira de Diagnóstico e Tratamento da Síndrome Metabólico (Carvalho et al., 2005)

A relevância que a SM tem para a saúde pública nos países em que os recursos econômicos são limitados é inquestionável (Dutra et al., 2012). Segundo o Adult Treatment Panel (ATP) III cholesterol guidelines, é recomendado avaliar e intervir nessa população com múltiplos fatores de risco para enfermidade cardiovascular, focando na prevenção primária (Fasanmade et al., 2013). Precisamente, com objetivo de identificar um método capaz de reduzir o uso de recursos, é que o estudo evidenciou um alto valor preditivo positivo de a CA para SM, ainda que, de forma individual, não encontrou associação 
de variáveis antropométricas com fatores de risco cardiovascular.

\section{Considerações Finais}

De forma geral, observou-se uma elevada prevalência dos fatores de risco para enfermidade cardiovascular, sendo que a circunferência abdominal foi um preditor significativo para a síndrome metabólica. Entretanto, algumas limitações existem devido ao caráter transversal da pesquisa. O corte transversal permitiu analisar efeito causal, porém, sujeito a apresentar viés de prevalência. Ainda assim, ressalta-se a importância das informações imediatas fornecidadas sobre a prevalência e fatores associados ao risco cardiovascular, as quais são essenciais para o planejamento de ações em saúde pública.

Tendo em vista que as políticas de governo para prevenir enfermidades cardiovasculares podem ser otimizados quando destinadas a prevenir e tratar a síndrome metabólica como um todo ao invés de seus fatores de risco individuais. Se recomenda utilizar a CA na prática clínica, um método simples e barato, acompanhada de parâmetros clínicos e exames de laboratórios para uma maior precisão diagnóstica de SM, eliminando possíveis falso-negativos e resultando em uma intervenção terapêutica adequada com menores gastos públicos futuros.

\section{Referências}

Carvalho, M. H. C. D., Mancini, M. C., Melo, M. E. D., Bahia, L. R., Negrão, C. E., Matos, L. D. N. J. D., \& Jardim, P. C. B. V. (2005). I Diretriz brasileira de diagnóstico e tratamento da síndrome metabólica. Arq Bras Cardiol, 84, suppl 1, 1-28.

Castro, S. H. D., Mato, H. J. D., \& Gomes, M. D. B. (2006). Anthropometric parameters and metabolic syndrome in type 2 diabetes. Arq Bras Endocrinol Metab, 50(3), 450-455.

Dutra, E. S., de Carvalho, K. M., Miyazaki, É., Merchán-Hamann, E., \& Ito, M. K. (2012). Metabolic syndrome in central Brazil: prevalence and correlates in the adult population. Diabetol Metab Syndr, 4(1), 1-9.

Fasanmade, O. A., Odeniyi, I. A., Amira, C. O., \& Okubadejo, N. U. (2013). Association of body mass index and abdominal adiposity with atherogenic lipid profile in Nigerians with type 2 diabetes and/or hypertension. Niger Med J, 54(6), 402.

Gierach, M., Gierach, J., Ewertowska, M., Arndt, A., \& Junik, R. (2014). Correlation between body mass index and waist circumference in patients with metabolic syndrome. ISRN Endocrinol, 2014, 514589.

Ginsberg, H. N., \& MacCallum, P. R. (2009). The obesity, metabolic syndrome, and type 2 diabetes mellitus pandemic: Part I. Increased cardiovascular disease risk and the importance of atherogenic dyslipidemia in persons with the metabolic syndrome and type 2 diabetes mellitus. J Cardiometab Syndr, 4(2), 113-119.

Glaner, M. F. (2005). Body mass index as indicative of body fat compared to the skinfolds. Rev Bras Med Esporte, 11, $243-246$.

Gomes, M. D. B., Giannella Neto, D., Mendonça, E. D., Tambascia, M. A., Fonseca, R. M., Réa, R. R., \& Dib, S. A. (2006). Nationwide multicenter study on the prevalence of overweight and obesity in type 2 diabetes mellitus in the Brazilian population. Arq Bras Endocrinol Metab, 50(1), 136-144.

Kodama, S., Horikawa, C., Fujihara, K., Heianza, Y., Hirasawa, R., Yachi, Y., \& Sone, H. (2012). Comparisons of the strength of associations with future type 2 diabetes risk among anthropometric obesity indicators, including waist-to-height ratio: a meta-analysis. Am J Epidemiol, 176(11), 959-969.

Marinho, N. B. P., Vasconcelos, H. C. A. D., Alencar, A. M. P. G., Almeida, P. C. D., \& Damasceno, M. M. C. (2012). Diabetes mellitus: associated factors among users of the family health strategy. Acta Paul Enferm, 25, 595-600.

Martins, K. A., Monego, E. T., Paulinelli, R. R., \& Freitas-Junior, R. (2011). Comparison of methods to evaluate total body fat and its distribution. Rev Bras Epidemiol, 14(4), 677-687.

Rocha, N. P. D., Siqueira-Catania, A., Barros, C. R., Pires, M. M., Folchetti, L. D., \& Ferreira, S. R. G. (2010). Analysis of several anthropometric measurements for the identification of metabolic syndrome, with or without disturbance of glucose metabolism. Arq Bras Endocrinol Metab, 54(7), 636-643.

Stolar, M. W., \& Chilton, R. J. (2003). Type 2 diabetes, cardiovascular risk, and the link to insulin resistance. Clinical therapeutics, 25 , B4-B31.

Van Dijk, S. B., Takken, T., Prinsen, E. C., \& Wittink, H. (2012). Different anthropometric adiposity measures and their association with cardiovascular disease risk factors: a meta-analysis. Neth Heart J, 20(5), 208-218. 